\title{
High Resolution S/TEM Study of Defects in MOCVD Grown Mono to Few Layer $\mathrm{WS}_{2}$
}

Saiphaneendra Bachu", Danielle Reifsnyder Hickey¹, Tanushree H Choudhury ${ }^{2}$, Mikhail Chubarov², Joan M Redwing ${ }^{1,2}$ and Nasim Alem ${ }^{1,2}$

1. Department of Materials Science and Engineering, The Pennsylvania State University, University Park, USA.

2. 2D Crystal Consortium, Materials Research Institute, The Pennsylvania State University, University Park, USA.

Two-dimensional (2D) Transition metal dichalcogenides (TMDCs) are interesting material systems for optoelectronic device applications due to their semiconducting behavior and their electronic structure transition from indirect to direct band gap upon thinning to a monolayer due to quantum confinement [1, 2]. While these materials systems have a strong potential to be incorporated in electronics and optoelectronics, the electronic properties of TMDs and thus their device performance strongly depends on the atomic structure of their defects (vacancies, dislocations and grain boundaries) [3].

To integrate the 2D TMDs into devices, it is also essential to synthesize them on large scale with uniform thickness. To date, majority of the devices made of 2D materials use exfoliated flakes rather than continuous films grown using controlled methods. Recently, monolayer $\mathrm{WSe}_{2}$ films have been grown using metalorganic chemical vapor deposition (MOCVD) with both metal and chalcogen precursors outside the reaction chamber to allow accurate control of their ratios and flow rates [4]. In this paper, we extend that work to MOCVD grown $\mathrm{WS}_{2}$ films and use high-resolution Scanning/Transmission Electron Microscopy (HR-S/TEM) to study the epitaxial nature and the resulting defect structure of the films. Figure 1a shows an optical image of the $\mathrm{WS}_{2}$ film, removed from the sapphire growth substrate, covering the holey carbon quantifoil grid over a large area. The grown $\mathrm{WS}_{2}$ is a coalesced monolayer film with triangles of few layer regions. A higher magnification TEM image and the corresponding diffraction pattern (DP) are presented in Figure 1b and 1c. The diffraction spots indicate the single orientation and thus the epitaxy of the grown film. Figure 1d is an Annular Dark Field STEM (ADF-STEM) image showing a grain boundary and the variation of number of layers on the top of one of those triangles. This presentation will further show the observed defect structures not only in single phase TMDs but also in the alloy TMD structures [5].

\section{References:}

[1] Jariwala, Deep et al, ACS nano 8.2 (2014), p. 1102.

[2] Zhao, Weijie et al, ACS nano 7.1 (2012), p. 791.

[3] Zhou, Wu et al, Nano letters 13.6 (2013), p. 2615.

[4] Zhang, Xiaotian et al, Nano letters (2018).

[5] This work was supported by National Science Foundation CAREER AWARD (NSF CAREER grant \#424-36 61X4). 

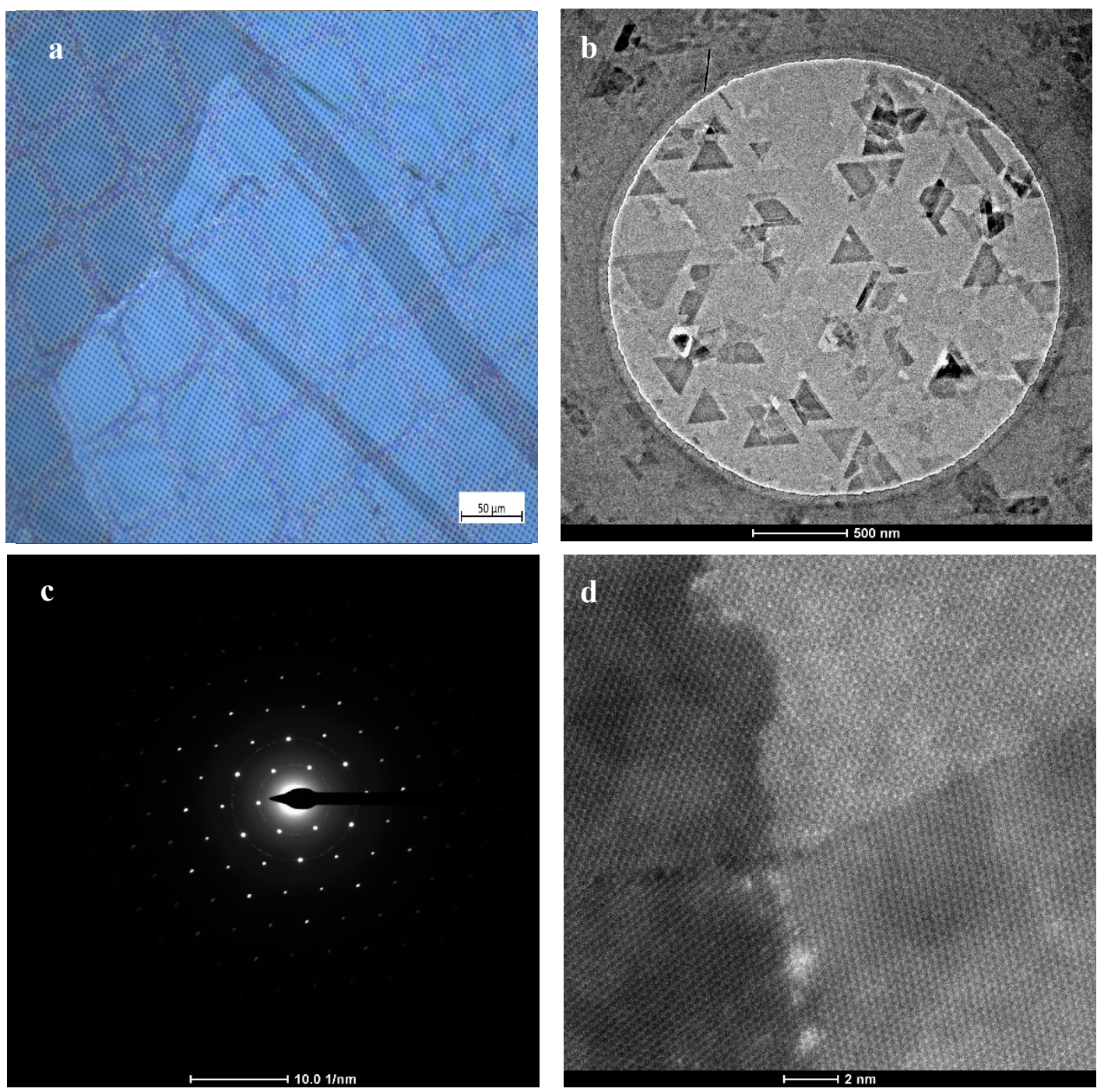

Figure 1 (a) Optical image of $\mathrm{WS}_{2}$ film covering the holey carbon grid, (b) and (c) HR-TEM image and the corresponding diffraction pattern and (d) an ADF-STEM image of a grain boundary going through regions of film with varying thickness 